\title{
Thread-based multiplexed sensor patch for real-time
} sweat monitoring

\author{
Trupti Terse-Thakoor ${ }^{1}$, Meera Punjiya ${ }^{1}$, Zimple Matharu ${ }^{1}$, Boyang Lyu ${ }^{1}$, Meraj Ahmad $\mathbb{D}^{2}$, Grace E. Giles ${ }^{3,4}$, Rachel Owyeung ${ }^{5}$, \\ Francesco Alaimo (iD), Maryam Shojaei Baghini ${ }^{2}$, Tad T. Brunyé ${ }^{3,4,6}$ and Sameer Sonkusale $\mathbb{D}^{1 凶}$
}

Sensor platforms that exploit the fibrous textile threads as substrates offer great promise since they can be directly sewn, woven or stitched on to any clothing. They can also be placed directly in intimate contact with the skin. In this work, we present a threadbased sensing platform in the form of a multiplexed sensing patch for continuous simultaneous on-skin monitoring of sweat. The patch performs real-time, on-body measurements of important biomarkers present in sweat such as electrolytes (sodium and ammonium ions), metabolites (lactate) and acidity ( $\mathrm{pH})$. Flexible threads coated with conductive inks were used as sensing electrodes. Selective potentiometric detection of electrolytes and $\mathrm{pH}$ was made possible through ion-selective membrane deposition and $\mathrm{pH}$-sensitive polyaniline coating on threads, respectively. An amperometric enzymatic sensing scheme with lactate oxidase was used for the detection of lactate. An array of the thread sensors is integrated onto a patch with connectivity to a miniaturized circuit module containing a potentiostat, microprocessor and wireless circuitry for wireless smartphone readout. Extensive in vitro validation and an in vivo human pilot study involving a maximal exertion test show the promise of this platform for real-time physiological monitoring of human performance/fitness under stress, as well as diagnostic monitoring through sweat analysis.

npj Flexible Electronics (2020)4:18; https://doi.org/10.1038/s41528-020-00081-w

\section{INTRODUCTION}

There is growing interest in health monitoring using minimally invasive wearable devices. Beyond biophysical markers such as electrocardiogram, respiration rate, and core temperature, there is interest in monitoring one's metabolic status from easy-to-sample biological fluids, such as sweat or saliva in real-time. Metabolic markers provide a more direct indicator of human health for accurate assessment of athletic performance, clinical diagnosis, and for managing chronic health conditions including generating alerts in cases of emergencies.

Among different choices of body fluids for continuous real-time monitoring, sweat is promising since it is easily accessible and can be collected noninvasively using the iontophoresis or chemical stimulation methods ${ }^{1,2}$. In addition, sweat is very rich in physiological and metabolic biomarkers, which are indicators of human health and performance and correlate well with blood plasma levels and therefore can serve as an ideal surrogate diagnostic fluid. For example sodium from sweat can indicate the hydration status and electrolyte imbalance in a body ${ }^{3-6}$, lactate concentration can be an indicator of the muscle fatigue ${ }^{7}$, chloride ion levels have been used in diagnosis of cystic fibrosis and cortisol, a stress hormone, can be used to assess emotional stress as well as metabolic and immune functions. Other markers in sweat include glucose for a person's energy level as well for diabetes monitoring, ammonium for metabolic conditions related to protein breakdown as result of diet $^{8}$, liver malfunction, or hypoxia. For more accurate diagnosis, one may need real-time noninvasive biosensors that can simultaneously detect multiple biomarkers indicative of underlying health condition. For example, athletes may want to monitor levels of all the electrolytes, hydration and any signs of muscular fatigue. Such levels may be informative during physical fitness tests, such as maximum oxygen uptake $\left(\mathrm{VO}_{2 \mathrm{max}}\right)$, as well as predicting performance peaks or declines during competition.

The most critical requirement for a sweat monitoring platform is its ability to do so in a minimally invasive manner, which we believe can be achieved using advances in flexible bioelectronics. For example, there have been recent reports of sweat sensors in different formats such as tattoos, sweatbands, wristbands, headbands $s^{1,2}$, which have been conveniently fabricated using tattoo paper $^{9-11}$, cellulosic paper substrate ${ }^{12}$, flexible polymers and combination of flexible polymers and solid-state integrated circuit (IC) devices. Though the sensors themselves are flexible, there have been numerous challenges to integrate electronics with them since electronics are inherently off-the-shelf hard components assembled on semirigid substrates. Moreover, many of the earlier platforms do not measure sweat analytes in real time due to the inability of continuous uptake and sampling of fresh sweat from the skin by the sensor platform. More recently, these issues have been resolved to a greater extent ${ }^{1,2}$. Of all the platforms developed so far, textile-based approaches provide the most versatility for sweat monitoring, since they can be integrated onto existing clothing. Moreover, textile-based platforms provide natural breathability to the underlying skin that promotes natural sweating and evaporative cooling which is not the case with most of the flexible polymeric substrates being used for sweat monitoring. In fact, fabric/textile based sweat sensors have been reported recently in the literature such as for the measurement of perspiration rate based on digital droplet flowmetry ${ }^{13}$, colorimetric sensing of $\mathrm{pH}$ and lactate ${ }^{14}$, and potentiometric sensing of

\footnotetext{
${ }^{1}$ Department of Electrical and Computer Engineering, Tufts University, Medford, MA 02155, USA. ${ }^{2}$ Department of Electrical Engineering, Indian Institute of Technology (IIT), Bombay, Mumbai 400076, India. ${ }^{3}$ Center for Applied Brain and Cognitive Sciences, Medford, MA 02155, USA. ${ }^{4}$ U.S. Army Combat Capabilities Development Command, Soldier Center, Natick, MA 01760, USA. ${ }^{5}$ Department of Chemical and Biological Engineering, Tufts University, Medford, MA 02155, USA. ${ }^{6}$ Tufts University, Department of Psychology, Medford, MA 02155, USA. ${ }^{\circledR}$ email: sameer@ece.tufts.edu
} 
a
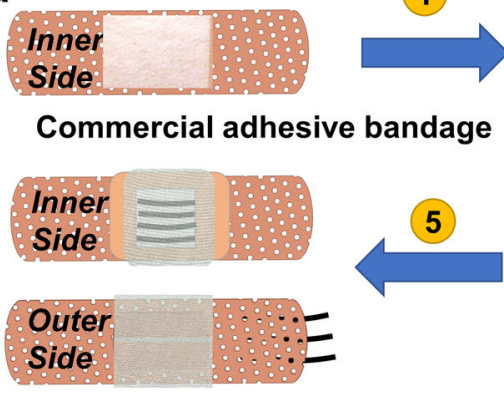

b

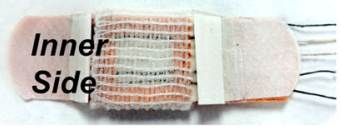

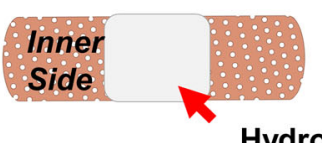

Hydrophobic adhesive film

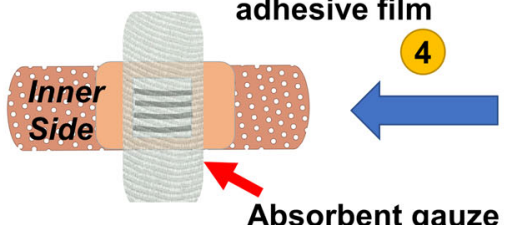

Absorbent gauze

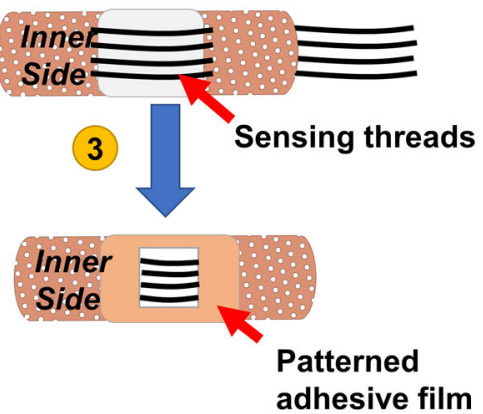

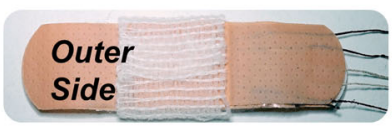

Fig. 1 Fabrication of thread-based sweat patch sensor. a Schematic of fabrication steps 1: adhesive hydrophobic film is placed on the center of the commercial adhesive bandage 2: sew thread sensors on the bandage 3: patterned adhesive film is placed to define the sensing zone 4: absorbent gauze is placed on the sensing zone 5: fold the gauze towards outer side of the bandage. b Photograph of the patch sensor prototype.

$\mathrm{pH}^{15}$. Even monitoring of continuous perspiration rate based on the changes in the conductivity between the conductive threads of a fabric has been reported ${ }^{16}$. However, these and other realizations of textile-based electrochemical sensors rely on screen-printing or dip-coating on-fabric resulting in nonuniformity and variability due to wicking/spreading of the ink on uneven textured textile surfaces. Moreover, screen-printing may not suitable for integration onto existing clothing (e.g., hats and gloves) after the clothing has already been made. Specialized custom approaches may be needed for uniform patterning of functional materials on to fabrics from solution ${ }^{17}$. We have recently proposed a bottom up approach using individual functionalized threads knitted/stitched together into a diagnostic platform $^{18,19}$. Our group reported development of a complete diagnostic platform where threads were used for monitoring of physiochemical tissue properties such as physical (strain) and chemical ( $\mathrm{pH}$, glucose) integrated with thread-based microfluidic networks and electronic interconnects ${ }^{18}$. We have also shown the applicability of these versatile threads in the form of patch for real time wound $\mathrm{pH}$ monitoring ${ }^{20,21}$ as well as on-demand drug delivery ${ }^{19}$. In these demonstrations, the self-wicking properties of threads have been used to realize passive pump-free microfluidics $^{22-24}$. There are also other reports on carbon nanotube infused thread electrodes for potentiometric sensing of potassium, ammonium, and $\mathrm{pH}^{25}$. Thread's inherent flexibility, tunable elasticity, strength, inertness, and controlled hydrophilic or hydrophobic properties make than an ideal substrate for wearable sensor platforms. Moreover, their universal availability and wellestablished processing is particularly useful especially in making a sweat sensing platform that can be integrated into any existing garment through stitching, sewing, or knitting. Typical on-fabric approaches proposed by other textile-based sensors do not allow for this kind of adaptability for personalized use cases. In this manuscript, we present a truly wearable fully integrated threadbased multiplexed platform in a band-aid format for real-time simultaneous detection of sodium, ammonium, $\mathrm{pH}$, and lactate directly from sweat with wireless electronic readout.

Our choice of sweat-based biomarker panel comprises of sodium, lactate, ammonium, and $\mathrm{pH}$. The markers were chosen judiciously to provide a real-time physiological measure of athletic performance and strenuous physical activity (e.g., in exercise or sports). As stated above, sodium can provide hydration status information, lactate concentration can indicate muscle fatigue (which can be monitored by both $\mathrm{pH}$ and lactate sensors), and ammonium provides an indication of anaerobic metabolism ${ }^{26,27}$.
To fabricate these sensing patches, we have used different threads such as those made from polyester (PE) and stainless steel (SS) threads coated with conductive inks as sensing electrodes. Selective potentiometric sensing of electrolyte and $\mathrm{pH}$ was achieved using a coat of polymeric ion-selective membrane and polyaniline, respectively. Amperometric sensing of metabolites was obtained using specific enzyme-coated threads. The sweat sensor platform was assembled as a patch with threads placed directly on the fabric gauze of the commercial bandage (Fig. 1). The fabric gauze facilitates sweat transport from the sensing area or inner side of the patch to the backside of the patch to evaporate. Gauze edges are carefully sealed to the patch and skin to ensure sweat does not evaporate from the sensing area, as that would result in ionic accumulation. Additional fabric gauze was used for sweat collection. Continuous sweat evaporation from the gauze ensures that fresh sweat is continually wicked at the sensor surface for real-time measurement. Threads are connected to reusable wireless readout electronics for real-time data acquisition and collection from potentiometric (for $\mathrm{pH}$, ammonium, and sodium sensing) as well as amperometric (for lactate) sensing.

\section{RESULTS}

\section{Characterization of threads}

To be able to use the threads as electrodes in electrochemical sensing, it is important that they are highly conductive. To obtain such highly conductive threads, PE threads were coated multiple times with conductive inks and characterized in terms of electrical conductivity. Figure 2 illustrates the fabrication steps to realize each thread type (vide infra). Figure 3a shows the resistance of the $\mathrm{PE}$ threads after each coating of carbon ink and $\mathrm{Ag} / \mathrm{AgCl}$ inks (C/PE and $\mathrm{Ag} / \mathrm{AgCl} / \mathrm{PE}$ threads, respectively). The first coating of inks drastically reduced the resistance, which plateaus after three coatings. We found that three layers of coating was optimal which resulted in the resistance of $115 \Omega / \mathrm{cm}$ for $C / P E$ and $0.5 \Omega / \mathrm{cm}$ for $\mathrm{Ag} / \mathrm{AgCl} / \mathrm{PE}$ threads. The conductive C/PE threads were further coated with ammonium and sodium ion selective polymeric membrane solutions for fabrication of ammonium (NH4/C/PE) and sodium ( $\mathrm{Na} / \mathrm{C} / \mathrm{PE})$ thread sensors, respectively. Whereas carbon coated SS threads (C/SS) were electrochemically deposited with polyaniline (PANI) for construction of $\mathrm{pH}$ sensors (PANI/C/SS). For lactate sensing thread (LOx/PB/PE) fabrication, prussian blue (PB) ink was coated on PE threads, followed by drop casting of lactate oxidase enzyme formulation. $\mathrm{Ag} / \mathrm{AgCl} / \mathrm{PE}$ threads were coated 
a

\section{Carbon Based Conductive Thread}

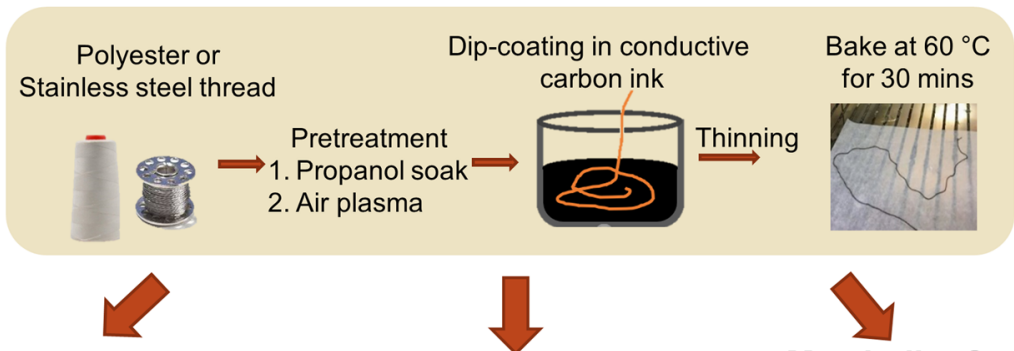

Electrolyte Sensing Thread

Dip-coating lonophore Solution

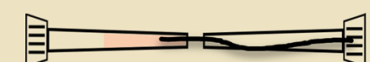

Pipette tip with

$\mathrm{NH}_{4}{ }^{+}$or $\mathrm{Na}^{+}$ ionophore solution
Pipette tip with Carbon thread
pH Sensing Thread

Electrochemical Deposition of PANI

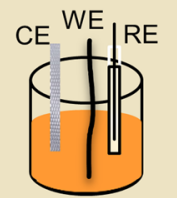

\section{Metabolite Sensing Thread}

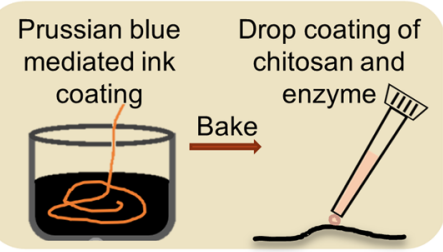

b

Silver-Silver Chloride Thread

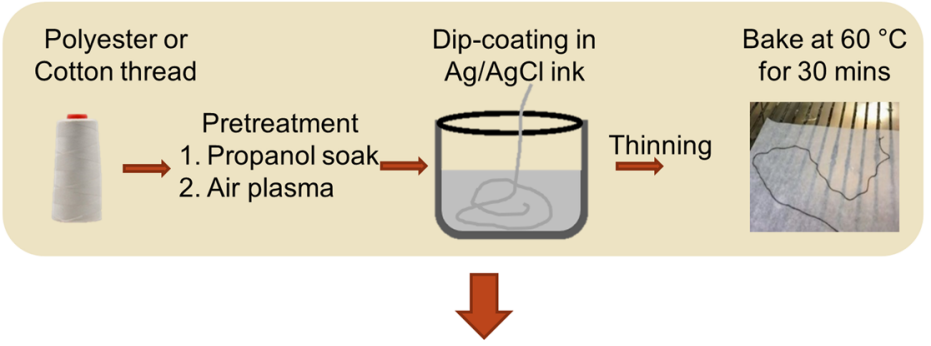

Solid-state Reference Thread

Dip-coating Solid-state Reference Membrane

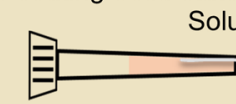

Pipette tip with

Polymeric reference

membrane solution

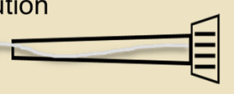

Pipette tip with $\mathrm{Ag} / \mathrm{AgCl}$ thread

Fig. 2 Construction of thread-based sensing and reference electrodes. Steps in preparation of a carbon-based conductive threads and sensing threads and $\mathbf{b}$ PVB-coated $\mathrm{Ag} / \mathrm{AgCl} / \mathrm{PE}$ thread.

with PVB cocktail solution, for their use as solid-state reference electrodes.

SEM characterization of bare conductive and sensing threads was also carried out. Figure 3b shows a SEM image of C/PE thread, partly scratched to show the inner thread fibrils and surrounding part showing the uniform carbon coating achieved after three coatings. An additional viewing angle of the C/PE thread can be found in Supplementary Fig. 1. Similarly, Fig. 3d shows the polymeric ammonium selective membrane coating and inner carbon layer coating on $\mathrm{NH} 4 / \mathrm{C} / \mathrm{PE}$ thread. Figure 3c, e shows the uniform PVB and PANI coating on $\mathrm{Ag} / \mathrm{AgCl}$ and C/PE threads, respectively.

\section{Performance of individual thread-based sensors}

The performance of each sensor was evaluated separately using standardized solutions of respective analytes. Figure $4 a-c$ shows open circuit potential responses of sodium, $\mathrm{pH}$, and ammonium sensors measured between ion-selective electrodes and reference electrode, using filter paper soaked in 1-1000 mM of $\mathrm{NaCl}, \mathrm{pH} 4-9$, and $0.1-100 \mathrm{mM}$ of $\mathrm{NH}_{4} \mathrm{Cl}$ solutions, respectively. The $\mathrm{NH} 4 / \mathrm{C} / \mathrm{PE}$, $\mathrm{Na} / \mathrm{C} / \mathrm{PE}$, and PANI/C/SS threads showed near-Nerstian behavior with sensitivities of $60.6,52.8$, and $62.3 \mathrm{mV}$ per decade of concentration of $\mathrm{NH}_{4}{ }^{+}, \mathrm{Na}+$, and $\mathrm{H}+$ ions, respectively. Chronoamperometric response of LOx/PB/PE sensor is shown in Fig. 4d within physiological relevant range and sensitivity of $900 \mathrm{nA} / \mathrm{mM}$. The response time of these sensors were calculated in dynamic conditions where different concentrations were added on the sensors. The response time was found to be in the range of 5-30 s, which seems appropriate for capturing the real-time changes in analyte concentration in sweat during physiological conditions. Supplementary Table 1 includes a comparison of our sensitivities with previous reports.

As the sweat concentration of analytes fluctuates over time, we also tested these sensors for hysteresis without any rinsing of the sensors. Reproducible OCP and current pattern with successive changes in ion (Fig. 5a-c) and lactate concentration (Fig. 5d) clearly demonstrates the viability of these sensors for real-time time sweat measurements. Moreover, it is suitable for single pre-/ post-calibration and validation during on-body human trials. The measurements provide an instantaneous value of the concentration of analyte in sweat, and does not account for the sweat rate, which can vary over an order of magnitude across subjects. One could utilize one of the published methods to measure sweat rate based on capillary action in future work ${ }^{28,29}$. 
a

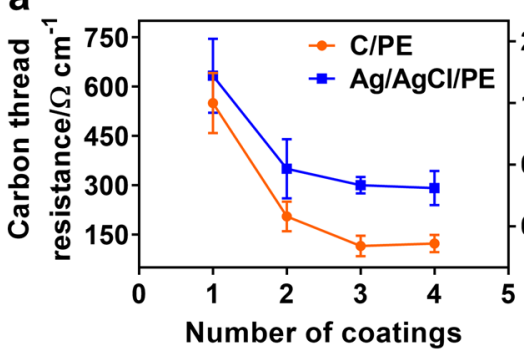

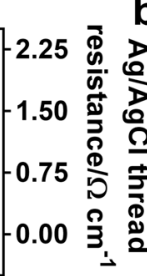

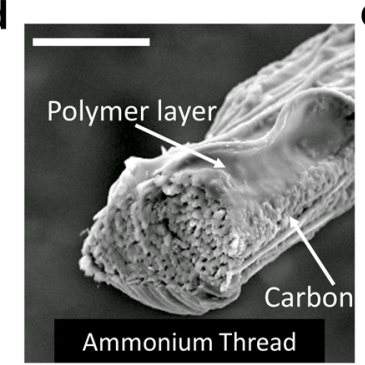

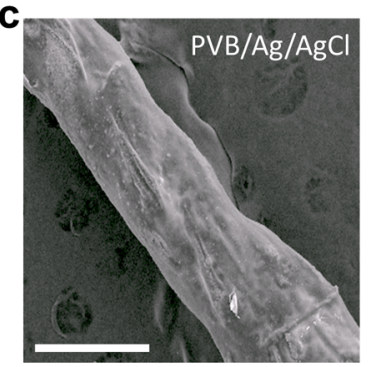

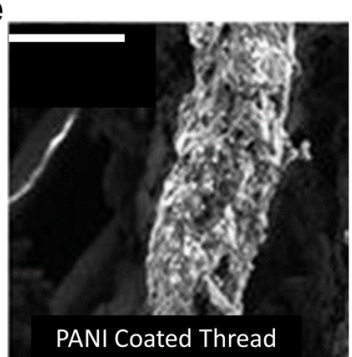

Fig. 3 Thread coatings characterization. a Electrical resistance $(\Omega / \mathrm{cm})$ of conductive carbon ink coated $\mathrm{PE}$ threads $(\mathrm{C} / \mathrm{PE})$ and $\mathrm{Ag} / \mathrm{AgCl}$ ink coated PE threads (Ag/AgCl/PE) after each coating cycle. Error bars from s.d. SEM images of b C/PE (scale bar $100 \mu \mathrm{m}$ ), c PVB/Ag/AgCl/PE (scale bar $500 \mu \mathrm{m}$ ), d NH4/C/PE (scale bar $200 \mu \mathrm{m}$ ), e PANI/C/PE (scale bar $10 \mu \mathrm{m}$ ) threads showing the surface coatings.
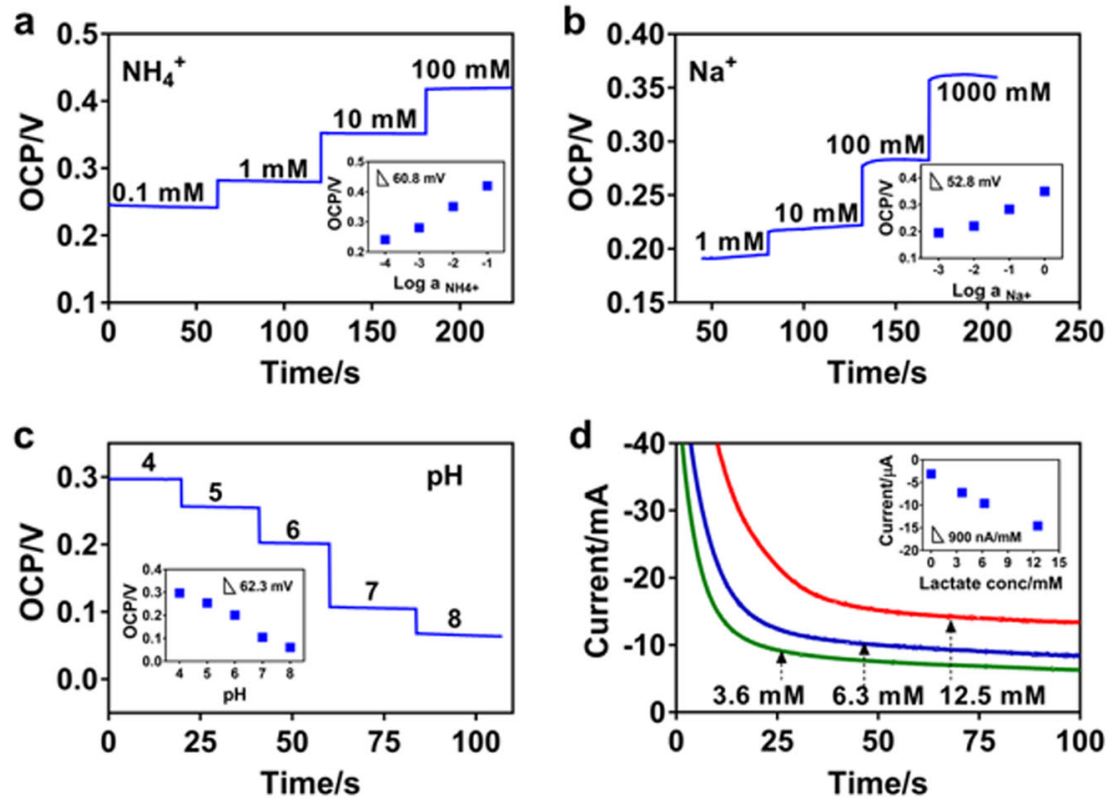

Fig. 4 Performance of thread-based sweat sensors. Potentiometric response of a ammonium, b sodium, $\mathbf{c} \mathrm{pH}$ and chronoamperometric response of $\mathbf{d}$ lactate using filter paper soaked in different concentration of respective analyte solution. Inset a-d shows the calibration plot of the respective thread sensors.

The selectivity of sweat sensors especially ion-selective electrodes is very important as sweat contains various electrolytes of similar charge and size. Supplementary Figure 2 shows that each thread sensor response negligibly to different interfering analytes compared to its specific analyte. The repeatability testing of each sensor also showed reproducible sensitivities (Supplementary Fig. 3). These sensors have also been tested for their drift in response over extended time of use (Supplementary Fig. 4). This clearly demonstrates that the thread-based sensor patch can be used for a minimum of $3 \mathrm{~h}$ without significant drift in sensor response. Additional characterization of the sensor reproducibility in real sweat measurements can be viewed in Supplementary
Fig. 5. Characterization of sensitivity reproducibility for $\mathrm{pH}$ and sodium sensors can be seen in Supplementary Fig. 6 .

Wireless readout electronics board

The primary metric for the electronics related to a wearable sweat sensing platform are the size, weight, area, and power consumption (SWAP). Individual circuit components should be chosen carefully to optimize the SWAP metric of the sensor system. From the measurement side, the AFE should have the ability to interface and measure current values from chronoamperometric measurement of lactate as well as the open circuit potential measurement for $\mathrm{pH}$, sodium, and ammonia sensing. We used a programmable, 

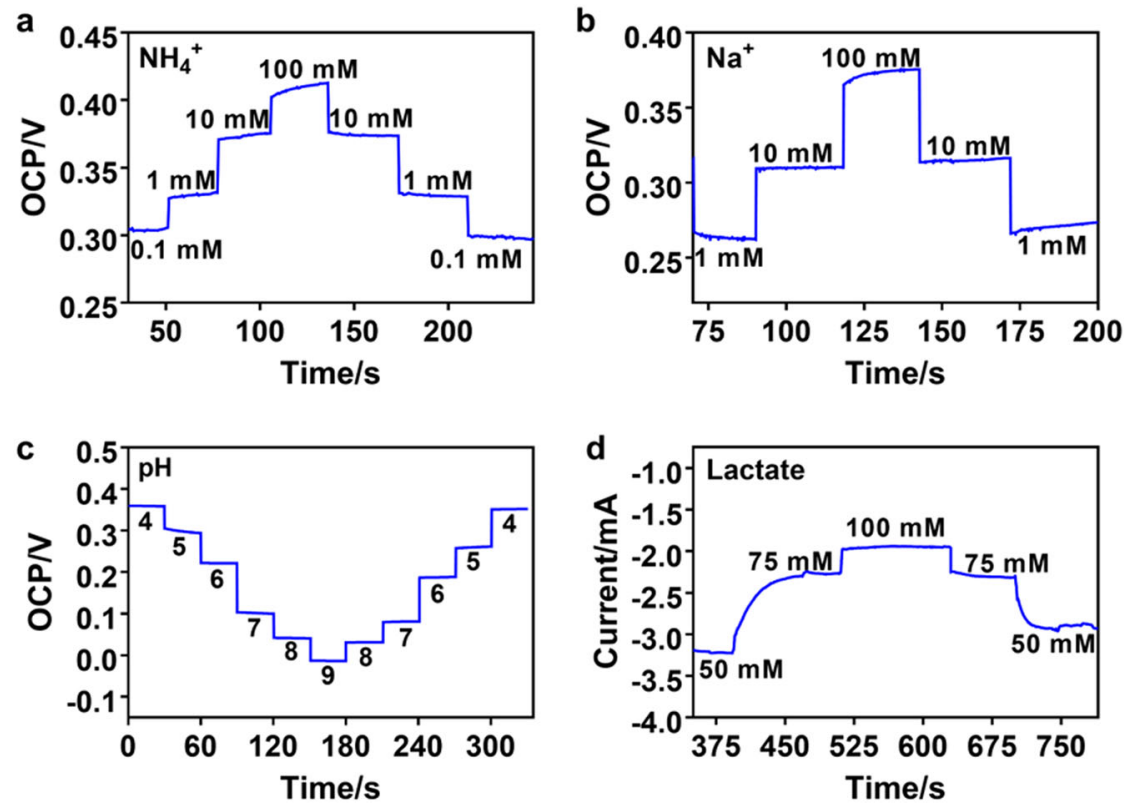

Fig. 5 Step response and hysteresis test of thread-based sweat sensors. Potentiometric response of a ammonium, b sodium, $\mathbf{c}$ pH, and chronoamperometric response of $\mathbf{d}$ lactate with changes in the analyte concentration without rinsing step.
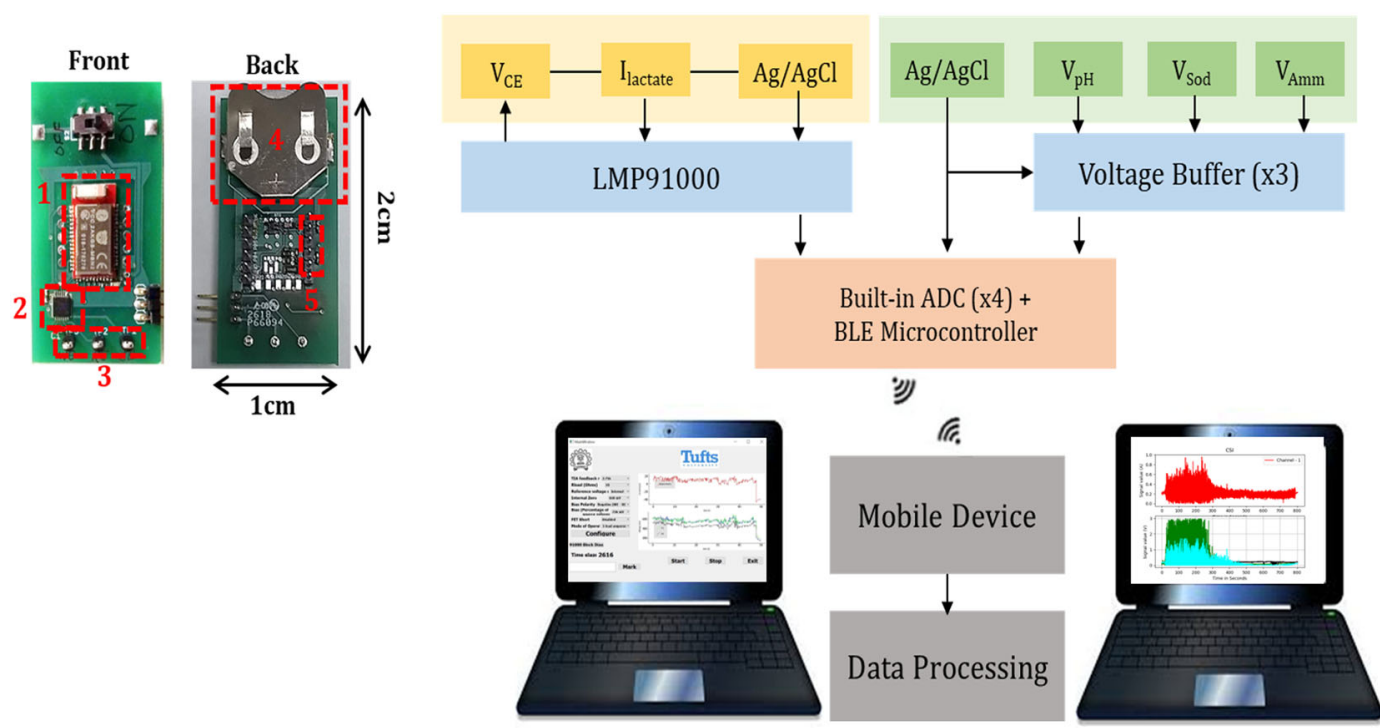

Fig. 6 Wireless readout electronics. Photograph of read out electronic where (1) RedBear BLE Module MB-N2 containing the Nordic semiconductor nRF52832 SoC and chip antenna, (2) LMP91000 configurable potentiostat, (3) amperometric connectors, (4) battery holder CR2032 (3.6 V lithium-ion) battery holder, (5) potentiometric connectors b block diagram of multiplexed sweat sensor patch read out electronics for potentiometric as well as amperometric measurements.

low power integrated circuit with capability to wirelessly configure the front end. A photograph of the developed system prototype is shown in Fig. 6. A graphical user interface (GUI), as shown in Supplementary Fig. 7, was developed for system programmability, data acquisition and data visualization. The stored data were then processed using Matlab and the converted to concentrations using calibration plots.

On-body sweat sensor patch trials on human volunteers

Initial on-body real-time sweat testing was carried out on four different healthy human subjects using individual sensor patch connected to a commercial potentiostat. Each working electrode for $\mathrm{NH}_{4}{ }^{+}, \mathrm{Na}^{+}$, and $\mathrm{pH}$ and reference electrode PVB/Ag/AgCl/PE were placed on a separate bandage for potentiometric measurement whereas $\mathrm{LOx} / \mathrm{PB} / \mathrm{C} / \mathrm{PE}$, thick $\mathrm{C} / \mathrm{PE}$, and reference electrode $\mathrm{PVB} / \mathrm{Ag} / \mathrm{AgCl} / \mathrm{PE}$ were placed on a separate bandage for amperomeric measurement of lactate. The threads sensors were connected to the leads of commercial potentiostat or those of the electronics board through wires with small IC clip/hooks. Sensors worked as expected under natural flexing and deformation during placement and use. One may need detailed studies if application warrants extreme mechanical deformation during use.

Figure 7a shows the application of the sensor patch on the upper arm of the subject. Before placing the sensors on the body, electrolyte sensors were conditioned for minimum for $30 \mathrm{~min}$ in the lowest analyte concentration $\left(0.1 \mathrm{mM}\right.$ for $\mathrm{NH}_{4}{ }^{+}$for $\mathrm{NH} 4 / \mathrm{C} / \mathrm{PE}$, 


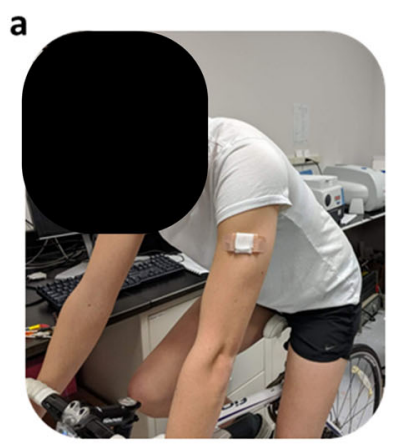

b

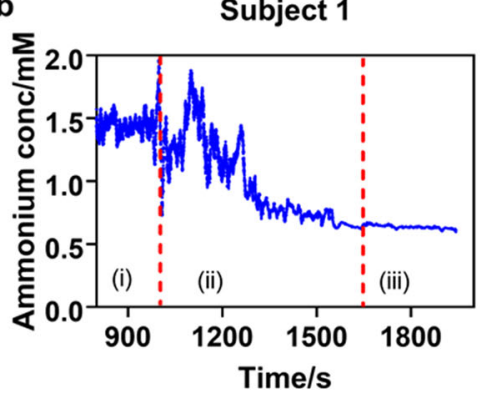

d

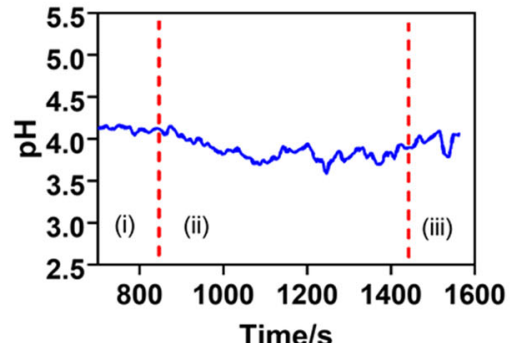

c

Subject 2

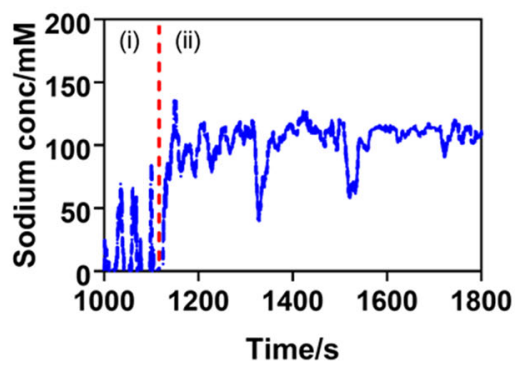

e

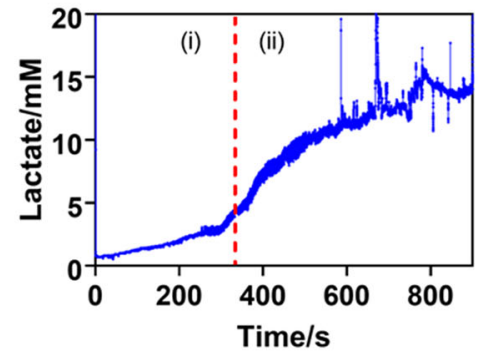

Fig. 7 Real-time sweat patch sensor data using benchtop potentiostat. a Photograph of a subject wearing the sweat sensor patch on the arm during the stationary cycling, real-time sensor data $\mathbf{b}$ ammonium, $\mathbf{c}$ sodium, $\mathbf{d} \mathbf{~ p H}$, and e lactate using a potentiostat from four different subjects. $\mathrm{i}$-iii in the plot represents equilibration, real-time measurement, and cool down period.

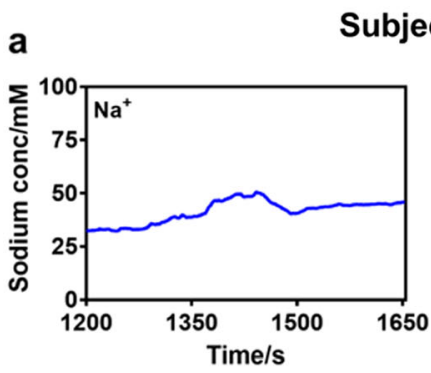

\section{Subject 5}
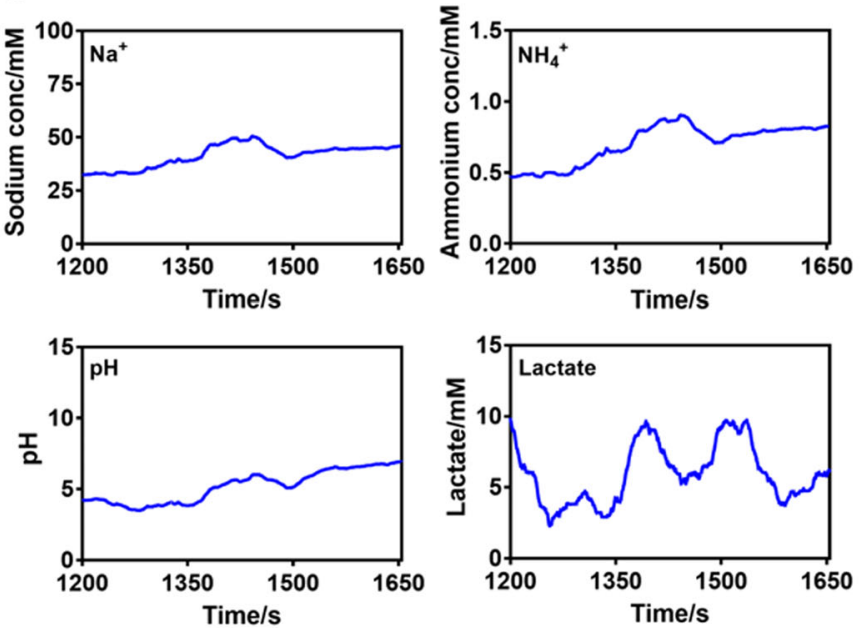

b
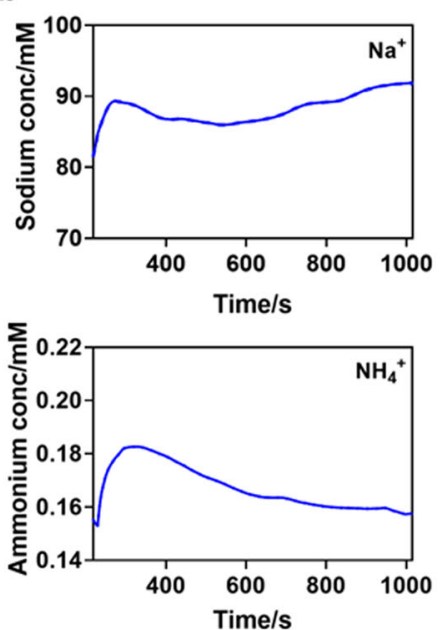

Fig. 8 Simultaneous and real-time multiplexed sweat patch sensor data using wearable readout electronics. a Sensor data of ammonium, sodium, $\mathrm{pH}$, and lactate of Subject 5 wearing the sweat sensor patch on the forehead during the stationary cycling. $\mathbf{b}$ Sensor data of Subject 6 wearing a waist belt integrated with sensor patch and the electronic during treadmill run.

$1 \mathrm{mM} \mathrm{Na}^{+}$for $\mathrm{Na} / \mathrm{C} / \mathrm{PE}$, and $\mathrm{PBKCl}$ for $\left.\mathrm{LOx} / \mathrm{PB} / \mathrm{C} / \mathrm{PE}\right)$. After conditioning, each sensor was calibrated for a minimum two times and then absorbent gauze was placed on the top of the patch sensor extending to the outer side of the patch to continuously wick away the sweat from the sensing area as shown in Fig. 2. The subjects then performed exercise on a stationary bike for minimum $30 \mathrm{~min}$ with intermittent sprinting, and the real-time sensing data were collected. The results of bike experiment are shown in Fig. 7b-d, which show real time sweat sensing data measured using a commercial potentiostat for $\mathrm{NH}_{4}{ }^{+}$, $\mathrm{Na}^{+}, \mathrm{pH}$, and lactate after subjects start sweating. Real sweat samples were collected from the volunteers during the tests to cross-check the on-body results with measurements performed separately. The results can be viewed in Supplementary Fig. 8. The plots represent the data corresponding to sensor equilibration, real time measurement, and cool down period. The sweat induction time (10-20 min) varies for each individual. During this time, the data with dry, floating electrodes is very noisy but as the sweat is induced the sensors get wet, equilibrate and generate more stable data. In Fig. 7 data collection, the electronics were not on-body. This resulted in noise likely due to the connections to the electronics being pulled as the user moved, not from the sensors themselves. We believe this to be the case as the sensors are isolated from the skin through the soft gauze. To reduce noise, we reinforced the connections of the threads into the custom electronics module with silver paste, to improve conductivity while maintaining flexibility. The electronics were also mounted to the same heart rate monitor, which significantly reduced noise, as 
shown in Fig. 8. Advanced algorithms based on canonical correlation analysis for artifact removal can also be employed in future realizations ${ }^{30}$.

We also conducted on-body sweat multiplexed sensing with readout using custom wireless readout electronics module during a maximal exertion test using a stationary bike (Subject 5) as well as treadmill run (Subjects 6 and 7). Figure 8a shows the multiplexed sweat sensing data of Subject 5 during the bike experiment. During this trial, wireless electronics was placed away from the subject (not directly on the body) to eliminate any motion-induced artifacts and connected to sweat patch using micro-hook connector wires. This resulted in significant noise in the data during sprinting. We resolved this problem in our treadmill run experiment by integrating the sweat patch and electronics together in a belt (Supplementary Fig. 9a) and resultant data can be seen in Fig. 8b (Subject 6) and Supplementary Fig. 10 (Subject 7). The sensing threads were also connected to electronics through DuPont female connectors and housings (Supplementary Fig. 9b, c). Threads can be connected to electronics board through simple knotting of silver wire (Supplementary Fig. 9d).

The $\mathrm{VO}_{2 \max }$ test resulted in $\mathrm{VO}_{2 \max }$ values of 43.8 and $54.6 \mathrm{ml} /$ $\mathrm{kg} / \mathrm{min}$ for Subjects 6 and 7, well above the United States average for females age $20-29(37.6 \pm 10.2 \mathrm{ml} / \mathrm{kg} / \mathrm{min})$. The data collected through on-body sensor patch trials could detect the real time changes in sweat $\mathrm{NH}_{4}{ }^{+}, \mathrm{Na}^{+}, \mathrm{pH}$, and lactate levels. We performed correlations between $\mathrm{VO}_{2}$ during the $\mathrm{VO}_{2 \max }$ test and analytes values beginning at $4 \mathrm{~min}$ for Subject 6 and at 6 min for Subject 7 . For Subject 6, higher $\mathrm{VO}_{2}$ was associated with higher sodium $(r=0.847, p<0.001)$ and lower ammonium $(r=-0.785$, $p=0.001)$. For Subject 7, higher $\mathrm{VO}_{2}$ was associated with higher $\mathrm{pH}(r=0.773, p=0.003)$ and lower ammonium $(r=-0.812$, $p=0.001)$, but not sodium $(p=0.064)$, see Supplementary Fig. 11. While one could try to infer individual's athletic fitness from the levels of biomarkers measured for each of the four Subjects, this is a very small sample study and is not the focus of this effort. Regardless, this demonstrates a preliminary feasibility study to investigate potential correlations between $\mathrm{VO}_{2 \max }$ and sweat analytics for real-time monitoring under vigorous exercise conditions. Even with a small sample set, results were consistent with expected performance given the individual's fitness and overall diet.

\section{DISCUSSION}

This paper reported thread-based multiplexed, electrochemical sensor including both potentiometric and amperometric sensors, realized on thread substrate, and packaged as a patch for realtime continuous monitoring of ammonium, $\mathrm{pH}$, lactate, and sodium levels in sweat. Beyond a patch, one can realize sweat sensing platform by stitching/sewing thread-based electrodes directly into any clothing (e.g., shirts and hats). See Supplementary Fig. 12 for some examples. The sensor patch was integrated with electronics for wireless connectivity for real-time data acquisition, storage, and processing. The performance of these thread-based sensors is comparable to other sensor substrates as well as standard ion-selective and amperometric sensor based on the sensitivity and selectivity. The sweat sensors detected changes in biomarkers associated with physical fitness and fatigue, and analyte levels changed as a function of oxygen uptake on a test of maximum oxygen uptake, the gold standard test of physical fitness. Further experiments are still required to extensively validate the sensors for real time quantitative measurements while also improving the sensor design itself such as making electronics more flexible and removing even residual motion artifacts using advanced signal processing.

The results presented in this paper were rigorously validated in vitro and in vivo with representative on-body trials. While the paper provides proof of feasibility, more on-body trials are necessary for accurate on-body statistical validation. This can be basis of future work (e.g., $\mathrm{VO}_{2 \max }$ exertion studies), the sensors can be statistically validated for their effectiveness in real-time continuous measurements of multiplexed biomarkers in the context of underlying clinical trials in lieu of sparse standardized measurements. We believe the thread-based sweat sensor platform has opened avenues for cost-effective, flexible wearable multiplexed monitoring of athletic fitness or health management.

\section{METHODS}

Materials

PE and smooth SS threads were purchased from local arts store and Sparkfun Electronics (Niwot, CO, USA), respectively. Carbon resistive ink (C-200) and silver-silver chloride (Ag/AgCl) (AGCL-675) inks were purchased from Applied ink solutions (Westborough, MA, USA). Prussian blue mediated carbon ink (C2070424P2) was procured from Gwent group, Sun chemicals Advanced materials (Parsippany, NJ, USA). ASTM type I deionized water, certified ACS grade were procured from fisher scientific (Hampton, NH USA). Lactate oxidase (50 KU, Toyobo, USA). Glucose oxidase from Aspergillis niger, ammonium chloride and sodium chloride of analytical grades, nonactin (ammonium ionophore), sodium ionophore $X$ (selectophore grade), 2-nitrophenyl octyl ether (o-NPOE), polyvinyl chloride (PVC), polyvinyl butyral (PVB), 2-nitrophenyl octyl ether (o-NPOE), sodium tetrakis[3,5-bis(trifluromethyl)phenyl] borate(Na-TFPB), bis(2-ethylhexyl) sebacate (DOS), tetrahydrofuran (THF), Aniline ACS grade, polyaniline emeraldine base, $\mathrm{MW} \sim 50,000$ (PANI) base were purchased from Sigma Aldrich (St. Louis, MO, USA). Sodium phosphate buffer $100 \mathrm{mM}$ of $\mathrm{pH} 7$ with $0.1 \mathrm{M} \mathrm{KCl}(\mathrm{PBKCl})$ was used in all the experiments otherwise mentioned. All the solutions for potentiometric measurements were prepared using ASTM type I deionized water.

\section{Instruments}

All ex situ electrochemical measurements were carried out using $760 \mathrm{E} \mathrm{CHI}$ electrochemical station analyzer. Potentiometric measurements involved a two-electrode set up of working and reference electrode. Conventional three-electrode set up of working, reference and counter electrode was used for amperometric measurements. Chronoamperometry was performed with initial voltage of $-0.06 \mathrm{~V}$ (based on the redox peaks of PB as shown in Supplementary Fig. 13), pulse width $1000 \mathrm{~s}$, quiet time $2 \mathrm{~s}$. Scanning electron microscope (SEM) images were obtained using Tescan VEGA3 Scanning Electron Microscope.

Preparation of conductive threads

PE threads were first cleaned by soaking them in isopropanol followed by air-drying. Threads were then treated with air plasma for $5 \mathrm{~min}$. This process removed wax used in commercial threads and rendered them hydrophilic so that conductive inks could infuse within the fibers of the threads. PE threads were infused and coated with carbon resistive and Ag/ $\mathrm{AgCl}$ inks to realize working thread i.e., C/PE electrode and reference electrode, i.e., $\mathrm{Ag} / \mathrm{AgCl} / \mathrm{PE}$, respectively. The ink-coated threads were cured at $60^{\circ} \mathrm{C}$ inside an oven for about $30 \mathrm{~min}$. SS conductive threads were used for making of the $\mathrm{pH}$ sensor. SS threads were first cleaned with IPA and then coated with carbon resistive ink, followed by baking at $60^{\circ} \mathrm{C}$ inside an oven for about $30 \mathrm{~min}$ to prepare C/SS thread. See Fig. 1 for graphical representation of these steps.

Preparation of ion-selective and solid-state reference solutions The ammonium ion-selective membrane cocktail solution was prepared by dissolving $0.4 \mathrm{mg}$ of nonactin, $138 \mathrm{mg}$ of o-NPOE, and $61.6 \mathrm{mg}$ of PVC in $2 \mathrm{ml}$ of THF. The sodium ion-selective membrane was prepared by dissolving $2 \mathrm{mg}$ of selectophore grade sodium ionophore $\mathrm{X}$, Na-TFPB, $66 \mathrm{mg}$ of PVC, and $130.9 \mathrm{mg}$ of DOS dissolved in $1.32 \mathrm{ml}$ of THF. Membrane cocktail solutions were thoroughly mixed by $30 \mathrm{~min}$ in an ultrasonic bath, sealed, and stored at $\sim 4^{\circ} \mathrm{C}$. $\mathrm{pH}$ or $\mathrm{H}^{+}$ion sensitive membrane solution was prepared by dissolving $500 \mathrm{mg}$ of PANI base in $100 \mathrm{ml}$ of hydrochloric acid ( $\mathrm{HCl}$ ) placed in a $\sim 4^{\circ} \mathrm{C}$ ice bath for $5 \mathrm{~h}$. Ink was transferred to a glass bottle, sealed and stored at $\sim 4{ }^{\circ} \mathrm{C}$ until usage. The aniline solution was prepared in sulphuric acid $\left(\mathrm{H}_{2} \mathrm{SO}_{4}\right)$ solution. The PVB cocktail solution for reference electrode was prepared by dissolving $78.1 \mathrm{mg}$ of PVB and $50 \mathrm{mg}$ of sodium 
chloride $(\mathrm{NaCl})$ into $1 \mathrm{ml}$ of methanol ${ }^{10}$. This mixture was thoroughly mixed by sonicating for around $30 \mathrm{~min}$ in an ultrasonic bath.

\section{Preparation of ammonium and sodium sensors}

$\mathrm{C} / \mathrm{PE}$ threads were coated with ammonium ion-selective membrane cocktail solution layer by layer using the dip-coating method. The four layers of membrane coating was optimized based on reproducibility of sensitivity. Ammonium sensor threads (NH4/C/PE) were then allowed to dry overnight and then stored at $4{ }^{\circ} \mathrm{C}$ until next use. Similar procedure was followed for sodium sensors except sodium ion-selective membrane solution was dip coated with three layers on C/PE threads to form sodiumsensing threads ( $\mathrm{Na} / \mathrm{C} / \mathrm{PE})$. See Fig. 1 for graphical representation of this process.

Preparation of solid-state reference thread electrodes

The solid-state reference membrane electrode (PVB/Ag/AgCl/PE) was prepared by dipping the $\mathrm{Ag} / \mathrm{AgCl} / \mathrm{PE}$ thread in the PVB cocktail solution and allowed to dry for an hour and used next day for measurement (as shown in Fig. 1).

\section{Preparation of $\mathrm{pH}$ sensors}

PANI was electrochemically deposited (chronopotentiometric method) on the surface of carbon-coated SS threads using Aniline solution for $70 \mathrm{~s}$ at anodic current of $150 \mu \mathrm{A}$ using an approach described earlier ${ }^{18,21}$. Conventional three-electrode setup was used whether C/SS thread was used a working electrode, double junction $\mathrm{Ag} / \mathrm{AgCl}$ as reference and platinum mesh electrode was used as a counter electrode. After the deposition, resulting functionalized thread was washed multiple times with deionized water to remove any aniline residues from the surface.

\section{Preparation of lactate sensors}

Prussian mediated ink was coated onto the PE threads and cured at $60^{\circ} \mathrm{C}$ inside an oven for about $30 \mathrm{~min}$ to form PB/PE threads. To define the active surface area, dielectric ink was coated on to the PB/PE threads. Chitosan $(1 \% \mathrm{~W} / \mathrm{v})$ solution was prepared in $0.1 \mathrm{M}$ acetic acid by magnetic stirring for $1 \mathrm{~h}$ at RT. Chitosan solution was mixed with PBKCl in 1:1 ratio. For lactate sensor (LOx/PB/PE), first diluted chitosan solution was drop casted onto the PB coated threads, dried and later coated with lactate oxidase $(45 \mathrm{mg} / \mathrm{ml}$ in $\mathrm{PBKCl}$ ) and again diluted chitosan solution. Enzyme coated threads were then stored at $4^{\circ} \mathrm{C}$ when not in use.

\section{Fabrication of patch sensor}

The sweat sensor was fabricated by assembling the sensing threads on to a commercial band-aid. See Fig. 2 for the detailed fabrication process flow. Where, adhesive hydrophobic film was first placed on the center of the band-aid and then working, and reference and counter threads were simply placed on it. To define the sensor area, which is important in amperometric measurements, dielectric ink was used to coat the thread as well as patterned insulating adhesive film was placed on the threads. The ends of threads are further coated with silver inks for better electrical contact with the readout electronics. Patterned absorbent gauze was used as a collection pad, placed over the threads and extended to the top side of the band-aid so that it is exposed to air for continuous evaporation. This results in continuous wicking of the sweat from the sensor surface, which enables real time sweat monitoring. While one can monitor the sweat rate by monitoring this evaporation process, that was not the focus of this work. Figure 2 shows the details of steps and an actual picture of a working sensing band-aid patch prototype.

\section{Wireless readout electronics board}

A Redbear Nano-2 microcontroller (SparkFun, Niwot, CO, USA) with Nordic Semiconductor nRF52832 SoC and chip antenna for bluetooth low energy was used for data acquisition and system programmability. The micropower integrated circuit LMP91000 from Texas Instruments (Dallas, TX) was used as an analog front end (AFE) for the chemical sensing applications. The IC LMP91000 was configured in three-lead electrochemical cell configuration, which eventually used for lactate sensing. The AFE is programmed via $12 \mathrm{C}$ communication using the Redbear Nano-2 microcontroller. The built-in 12-bit analog-to-digital converter (ADC) was used to acquire and measure the potential to measure the concentration of $\mathrm{pH}$, sodium, and ammonia. A voltage buffer is added between LMP91000 and the microcontroller to avoid electrical loading from the microcontroller. A rechargeable battery of $3.6 \mathrm{~V}$ (LIR2032) is used. A low dropout regulator from Texas Instrument, TLV70433 was used to generate a stable $3.3 \mathrm{~V}$ power supply for all the chips on the board including the LMP91000 and the RedBear. Also, since we do not expect to measure analytes any faster than once every second, we chose the sampling rate for data acquisition at $10 \mathrm{~Hz}$ which is more than adequate.

\section{Characterization of $\mathrm{pH}$, ammonium, and sodium sensors}

For potentiometric measurements of $\mathrm{pH}$, ammonium and sodium, two electrode set up was used where $\mathrm{NH} 4 / \mathrm{C} / \mathrm{PE}$ and $\mathrm{Na} / \mathrm{C} / \mathrm{PE}$ threads were used as working electrodes and $\mathrm{PVB} / \mathrm{Ag} / \mathrm{AgCl} / \mathrm{PE}$ thread was used as the reference electrode. Before being used for the first time, conditioning of the membrane-coated threads was carried out using the lowest concentration of respective analyte solution for a minimum of $30 \mathrm{~min}$ at room temperature. Before each sensor calibration, ends of the working and reference threads were connected to the commercial potentiostat. The different concentration solutions of respective analyte were prepared in deionized water. The filter paper was cut to the size such that it covers both working and reference electrode. The filter paper was placed onto the working and reference electrode and $100 \mu \mathrm{L}$ of lowest concentration solution was dropped onto the filter paper. The sensor was allowed to equilibrate for $60 \mathrm{~s}$ before taking measurements. Open circuit potentials (OCPs) between WE and RE were recorded as a function of time. After the measurement, filter paper was removed, and fresh filter paper was placed and repeated the earlier procedure with solution of increasing concentration. The hysteresis behavior of the sensor was also studied by testing the successive concentration of respective analyte. Selectivity of the ISE sensors was determined by testing the target analyte in presence of other interfering electrolytes normally present in sweat such as sodium, ammonium, and potassium.

\section{Characterization of lactate sensor}

For amperometric measurement of lactate, a three-electrode set up was used where $\mathrm{LOX} / \mathrm{PB} / \mathrm{C} / \mathrm{PE}$ threads used as working electrodes, thick $\mathrm{C} / \mathrm{PE}$ thread as the counter electrode and $\mathrm{PVB} / \mathrm{Ag} / \mathrm{AgCl} / \mathrm{PE}$ as a reference electrode. $\mathrm{PB} / \mathrm{C} / \mathrm{PE}$ threads and $\mathrm{LOx} / \mathrm{PB} / \mathrm{C} / \mathrm{PE}$ threads were first characterized by performing cyclic voltammetry measurements in PBKCL using commercial potentiostat. Next cyclic voltammetry measurements of lactate sensor were performed in the presence of lactic acid solutions in $\mathrm{PBKCl}$ to identify the hydrogen peroxide $\left(\mathrm{H}_{2} \mathrm{O}_{2}\right)$ reduction peak potentials. Chronoamperometric measurement were performed with filter paper soaked in increasing lactate concentration $(2-100 \mathrm{mM})$. The selectivity of lactate sensors was determined in presence of other metabolites i.e glucose as well as electroactive compounds such as ascorbic acid and uric acid at concentrations normally present in the sweat. The sensor performance is not expected to have temperature dependence since the sensor surface is expected to be close to the body temperature which is highly regulated at $37^{\circ} \mathrm{C}$ in normal individuals, one does need to conduct rigorous validation against temperature fluctuations which could be basis of the follow on work.

\section{On-body trials of sweat sensing patch in human volunteers}

On-body in vivo trials were first carried out on healthy male and female volunteers of age group of 22-35 years. The study group consisted of both physically active individuals who exercised regularly including strength training and consumed moderate to high protein diet, and other individuals with little or no exercise and consumed mixed diet with no strict dietary regulations. Information on the perspiration patterns of each subject was noted and placement of the sensor patch on the body was decided based on this information. Patches were placed on either the lower back, forehead or the arms of the different subjects. Before placing the sensors, the area of application was cleaned with alcohol wipes. The subjects performed minimum of $30 \mathrm{~min}$ of exercise on a stationary bike with intermittent sprinting or increase in resistance. The bike session consisted of initial ramp up to induce the sweat secretion then constant speed and in between sprinting of $1 \mathrm{~min}$. Toward the end of the session, there was a cool down period and then a complete stop. The first sets of trials were carried out with patch consisting of single analyte sensing threads separately and real-time measurement was done using a commercial potentiostat. 
A second set of in vivo trials used a maximal exertion test, and was carried out with a patch consisting of multiple analyte sensing threads and simultaneous detection using wireless readout electronics. Two participants were female, age 22-25 years, and regular runners who ran 40-43 miles per week and engaged in at least $30 \mathrm{~min}$ of moderateintensity physical activity 6 days per week. Participants were not pregnant or nursing, did not use illicit drugs, excessive alcohol and/or performance enhancing drugs, and did not have any orthopedic injuries or asthma. Patches were applied in the same manner as described above. Participants then completed maximal oxygen consumption $\left(\mathrm{VO}_{2 \max }\right)$ testing using a modified version of the protocol used in ${ }^{31}$. Participants were fitted with a heart rate monitor, mouthpiece, and headgear to collect respiratory gases for analysis. They then jogged for three minutes at five miles per hour (MPH) at a $1 \%$ grade. After $3 \mathrm{~min}$, the treadmill speed was set at $5.5 \mathrm{MPH}$ and increased by $0.2 \mathrm{MPH}$ every $30 \mathrm{~s}$, with the grade fixed at $1 \%$. When participants could no longer physically continue, they volitionally terminated testing by placing their hands and feet on the support rails located along both sides of the treadmill. At this point the treadmill speed was reduced to a slow walk. All participants performed a low-intensity cool-down for no less than two minutes. The criteria for reaching $\mathrm{VO}_{2 \max }$ included a plateau in $\mathrm{VO}_{2}<2 \mathrm{ml} / \mathrm{kg} / \mathrm{min}$ with an increase in workload, surpassing 1.10 respiratory exchange ratio, or achieving a peak heart rate at or above $95 \%$ of age-predicted maximum heart rate (220-age), or reporting a rating of perceived exertion great than or equal to 17 (i.e., "very hard"). $\mathrm{VO}_{2 \max }$ was calculated as the average of the three highest, continuous 20-s interval $\mathrm{VO}_{2}$ measurements. In both sets of trials the sensing threads were precalibrated with single as well as two-point calibration.

Written informed consent was obtained, and both the Tufts University Institutional Review Board and the Army Human Research Protections Office approved all procedures. Photographs used were obtained with subject's consent.

\section{DATA AVAILABILITY}

The data sets generated during and/or analyzed during the current study are available from the corresponding author on reasonable request.

Received: 9 October 2019; Accepted: 3 July 2020;

Published online: 28 July 2020

\section{REFERENCES}

1. Bariya, M., Nyein, H. Y. Y. \& Javey, A. Wearable sweat sensors. Nat. Electron. 1 160-171 (2018)

2. Choi, J., Ghaffari, R., Baker, L. B. \& Rogers, J. A. Skin-interfaced systems for sweat collection and analytics. Sci. Adv. 4, eaar3921 (2018).

3. Shirreffs, S. M. \& Sawka, M. N. Fluid and electrolyte needs for training, competition, and recovery. J. Sports Sci. 29, S39-S46 (2011).

4. Shirreffs, S. M., Armstrong, L. E. \& Cheuvront, S. N. Fluid and electrolyte needs for preparation and recovery from training and competition. J. Sports Sci. 22, 57-63 (2004).

5. Hoffman, M. D., Hew-Butler, T. \& Stuempfle, K. J. Exercise-associated hyponatremia and hydration status in 161-km ultramarathoners. Med. Sci. Sports Exerc. 45, 784-791 (2013)

6. Hoffman, M. D., Stuempfle, K. J., Rogers, I. R., Weschler, L. B. \& Hew-Butler, T. Hyponatremia in the $2009161-\mathrm{km}$ western states endurance run. Int. J. Sports Physiol. Perform. 7, 6-10 (2012).

7. Robergs, R. A., Ghiasvand, F. \& Parker, D. Biochemistry of exercise-induced metabolic acidosis. Am. J. Physiol. Regul. Integr. Comp. Physiol. 287, R502-R516 (2004).

8. Snow, R. J., Carey, M. F., Stathis, C. G. \& Febbraio, M. A. Effect of carbohydrate ingestion on ammonia metabolism during exercise in humans. J. Appl. Physiol. $\mathbf{8 8}$, 1576-1580 (2000).

9. Bandodkar, A. J. et al. Epidermal tattoo potentiometric sodium sensors with wireless signal transduction for continuous non-invasive sweat monitoring. Biosens. Bioelectron. 54, 603-609 (2014).

10. Guinovart, T., Bandodkar, A. J., Windmiller, J. R., Andrade, F. J. \& Wang, J. A potentiometric tattoo sensor for monitoring ammonium in sweat. Analyst 138, 7031-7038 (2013).

11. Jia, W. Z. et al. Electrochemical tattoo biosensors for real-time noninvasive lactate monitoring in human perspiration. Anal. Chem. 85, 6553-6560 (2013).
12. Koh, A. et al. A soft, wearable microfluidic device for the capture, storage, and colorimetric sensing of sweat. Sci. Transl. Med. 8, 1-23 (2016).

13. Yang, Y. H. et al. Wearable microfluidics: fabric-based digital droplet flowmetry for perspiration analysis. Lab Chip 17, 926-935 (2017).

14. Promphet, N. et al. Non-invasive textile based colorimetric sensor for the simultaneous detection of sweat pH and lactate. Talanta 192, 424-430 (2019).

15. Zamora, M. L. et al. Potentiometric textile-based pH sensor. Sens Actuators $B$ Chem. 260, 601-608 (2018).

16. Jia, J. et al. Conductive thread-based textile sensor for continuous perspiration level monitoring. Sensors 18, 3775 (2018).

17. Takamatsu, S. et al. Direct patterning of organic conductors on knitted textiles for long-term electrocardiography. Sci. Rep. 5, 15003 (2015).

18. Mostafalu, P. et al. A toolkit of thread-based microfluidics, sensors, and electronics for 3D tissue embedding for medical diagnostics. Microsyst. Nanoeng. 2, 16039 (2016).

19. Mostafalu, P. et al. A textile dressing for temporal and dosage controlled drug delivery. Adv. Funct. Mater. 27, 1702399 (2017).

20. Mostafalu, P. et al. Smart bandage for monitoring and treatment of chronic wounds. Small 14, 1703509 (2018).

21. Punjiya, M., Nejad, H. R., Mostafalu, P. \& Sonkusale, S. pH sensing threads with CMOS readout for smart bandages. in Proc 2017 IEEE International Symposium on Circuits (2017).

22. Li, X., Tian, J. F. \& Shen, W. Thread as a versatile material for low-cost microfluidic diagnostics. ACS Appl. Mater. Interfaces 2, 1-6 (2010).

23. Ballerini, D. R., Li, X. \& Shen, W. Flow control concepts for thread-based microfluidic devices. Biomicrofluidics 5, 14105 (2011).

24. Safavieh, R., Zhou, G. Z. \& Juncker, D. Microfluidics made of yarns and knots: from fundamental properties to simple networks and operations. Lab Chip 11, 2618-2624 (2011).

25. Guinovart, T., Parrilla, M., Crespo, G. A., Rius, F. X. \& Andrade, F. J. Potentiometric sensors using cotton yarns, carbon nanotubes and polymeric membranes. Analyst 138, 5208-5215 (2013).

26. Alvear-Ordenes, I., Garcia-Lopez, D., De Paz, J. A. \& Gonzalez-Gallego, J. Sweat lactate, ammonia, and urea in rugby players. Int. J. Sports Med. 26, 632-637 (2005).

27. Mitsubayashi, K., Suzuki, M., Tamiya, E. \& Karube, I. Analysis of metabolites in sweat as a measure of physical condition. Anal. Chim. Acta 289, 27-34 (1994).

28. Jain, V., Ochoa, M., Jiang, H., Rahimi, R. \& Ziaie, B. A mass-customizable dermal patch with discrete colorimetric indicators for personalized sweat rate quantification. Microsyst. Nanoeng. 5, 29 (2019).

29. Bandodkar, A. J. et al. Battery-free, skin-interfaced microfluidic/electronic systems for simultaneous electrochemical, colorimetric, and volumetric analysis of sweat. Sci. Adv. 5, eaav3294 (2019).

30. De Clercq, W., Vergult, A., Vanrumste, B., Van Paesschen, W. \& Van Huffel, S. Canonical correlation analysis applied to remove muscle artifacts from the electroencephalogram. IEEE Trans. Biomed. Eng. 53, 2583-2587 (2006).

31. Lourenco, T. F., Barreto Martins, L. E., Tessutti, L. S., Brenzikofer, R. \& Macedo, D. V. Reproducibility of an incremental treadmill $\left.\mathrm{VO}_{2}\right)$ max test with gas exchange analysis for runners. J. Strength Cond. Res. 25, 1994-1999 (2011).

\section{ACKNOWLEDGEMENTS}

All authors acknowledge the support from the Center for Applied Brain and Cognitive Sciences (CABCS), a U.S. Army Combat Capabilities Development Command, Soldier Center (Natick, MA) under Cooperative Agreement W911QY-15-2-0001. S.S also acknowledges the partial support of the Office of Naval Research (ONR) grant N001416-1-2550. M.S.B. and S.S. would also like to acknowledge the support from DST (Department of Science and Technology) and MHRD (Ministry of Human Resource Development), Government of India, under the SPARC (Scheme for the Promotion of Academic and Research Collaboration).

\section{AUTHOR CONTRIBUTIONS}

S.S. conceived the idea of thread-based sensing and diagnostics and its application for sweat monitoring. He also helped in the design of the platform and supervised the overall project. T.T. is a lead author who provided major contribution in the conceptualization, design, fabrication, test, validation of the sensor platform, and for performing on-body patch trials. M.P. and B.L. helped in the realization of sensors and electronics. Z.M. performed initial studies on sensing chemistry on threads. M.A. helped in the realization of electronics module and the graphical user interface (GUI) for wireless readout. M.S.B. and S.S. supervised the effort on readout electronics and GUI. R.O. helped in on-body sensor trials and formatting graphical figures and writing of the paper. F.A. assisted in coating threads with different inks. G.G. supervised the 
integration of sweat sensor application with maximum oxygen uptake testing and evaluated associations between human performance and sweat sensor data. T.B. supervised the overall effort with S.S. through problem definition and execution, suggested the sweat biomarker panel in the context of stress/fatigue monitoring, and provided critical paper feedback.

\section{COMPETING INTERESTS}

The authors declare no competing interests.

\section{ADDITIONAL INFORMATION}

Supplementary information is available for this paper at https://doi.org/10.1038/ s41528-020-00081-w.

Correspondence and requests for materials should be addressed to S.S.

Reprints and permission information is available at http://www.nature.com/ reprints
Publisher's note Springer Nature remains neutral with regard to jurisdictional claims in published maps and institutional affiliations.

(i) Open Access This article is licensed under a Creative Commons Attribution 4.0 International License, which permits use, sharing, adaptation, distribution and reproduction in any medium or format, as long as you give appropriate credit to the original author(s) and the source, provide a link to the Creative Commons license, and indicate if changes were made. The images or other third party material in this article are included in the article's Creative Commons license, unless indicated otherwise in a credit line to the material. If material is not included in the article's Creative Commons license and your intended use is not permitted by statutory regulation or exceeds the permitted use, you will need to obtain permission directly from the copyright holder. To view a copy of this license, visit http://creativecommons. org/licenses/by/4.0/.

(c) The Author(s) 2020 